\section{Bridging-to-transplant with azacitidine for myelodysplastic syndrome and acute myeloid leukemia, reduces the incidence of acute graft-versus-host disease}

\author{
Koichi Murakami, Hironori Ueno, \\ Takashi Okabe, Toshiya Kagoo, \\ Saigen Boku, Takahiro Yano, \\ Akihiro Yokoyama
}

Division of Hematology, Department of Internal Medicine, National Hospital Organization Tokyo Medical Center, Tokyo, Japan

\begin{abstract}
Allogeneic stem cell transplantation (allo-SCT) is the only curative option for myelodysplastic syndrome (MDS) and acute myeloid leukemia (AML). Azacitidine (AZA) has a good toxicity profile compared with intensive chemotherapy and can be considered a pre-transplant regimen in elderly patients and in patients with comorbidities. To investigate the impact of pre-transplant AZA on patient outcome after allo-SCT, we conducted a retrospective analysis of AZA pre-treatment followed by allo-SCT in patients with high-risk MDS and AML. Twenty patients who were divided into two groups according to AZA treatment given prior to allo-SCT (AZA vs nonAZA group, 10 each). Overall survival, event-free survival and incidence of chronic graft-versus-host disease (GVHD) were not significantly different between the two groups. The overall incidence of grade II to IV acute GVHD in the AZA group was significantly lower than that in the non-AZA group $(\mathrm{P}=0.004)$. Bridging to transplant with AZA should be considered as an immunomodulator and effective treatment strategy for patients with MDS and AML.
\end{abstract}

\section{Introduction}

At present, allogeneic stem cell transplantation (allo-SCT) is the only curative approach available for myelodysplastic syndrome (MDS) and acute myeloid leukemia (AML) patients, with poor prognostic factors. ${ }^{1}$ Allo-SCT can also prolong survival of the elderly patients aged 60-70 years with high-risk MDS compared to chemotherapy alone. ${ }^{2}$ However, pre-allo-SCT intensive induction chemotherapy (ICT) is often associated with adverse events that could eventually preclude SCT, or may lead to a worse SCT outcome., ${ }^{1,3}$ Thus, which treatment should be administered before transplantation is still controversial. Recently, azacitidine (AZA), a DNA demethylating agent, has emerged as a new agent used in a pre-transplantation conditioning therapy, but its clinical impact on transplantation remains unknown. According to the International Prognostic Scoring System (IPSS), AZA, with less toxicity than conventional induction chemotherapy, significantly prolongs overall survival of intermediate- 2 and high-risk MDS patients. ${ }^{4}$ Furthermore, induction and post-remission therapy using AZA may contribute to a longer survival in elderly patients with AML. 5,6 On the other hand, AZA can upregulate the expression of putative tumor antigens on leukemic blasts in vitro, induce a CD8+ $\mathrm{T}$ cell response, and expand the number of immunomodulatory T-regulatory cells (Tregs), suggesting a potential mechanism for augmenting graft-versus-leukemia (GVL) effect without increasing graft-versus-host disease (GVHD). ${ }^{7,8} \mathrm{We}$, therefore, retrospectively analyzed treatment outcome of patients who have received allo-SCT from April 2012 to July 2014 at our institution with or without AZA pre-transplantation therapy, mainly focusing on the incidence and severity of acute GVHD.

\section{Materials and Methods}

Characteristics of participating patients are shown in Table 1. All twenty patients were divided into two groups according to AZA treatment given prior to allo-SCT (AZA vs non-AZA group, 10 each). The AZA group consisted of 6 men and 4 women, while the non-AZA group had 7 men and 3 women. The median age at transplantation of the AZA group and the nonAZA group was 53 years (32-66) and 52.5 years (29-65) respectively. AZA was scheduled to be administered subcutaneously or intravenously at $75 \mathrm{mg} / \mathrm{m}^{2} /$ day for 7 days every 28 days. Patients in the AZA group had received a median of 3.5 cycles (range, 1-6 cycles) of AZA therapy before alloSCT. As main conditioning regimen for allo-SCT, patients in the AZA group received fludarabine $(\mathrm{Flu})$, busulfan $(\mathrm{Bu})$, and total body irradiation (TBI) $(n=3)$, $\mathrm{Bu} /$ cyclophosphamide (Cy) $(\mathrm{n}=3)$, and $\mathrm{Cy} / \mathrm{TBI}(\mathrm{n}=2)$, and patients in the non-AZA group received Flu/melphalan/TBI $(n=4)$ and $\mathrm{Cy} / \mathrm{TBI}(\mathrm{n}=2)$. GVHD prophylaxis consisted of cyclosporine with short-term methotrexate (sMTX) for HLA-matched-
Correspondence: Akihiro Yokoyama, Division of Hematology, Department of Internal Medicine, National Hospital Organization Tokyo Medical Center, 2-5-1, Higashigaoka, Meguro-ku, Tokyo, Japan.

Tel.: +81.33411.0111 - Fax: +81.33411.0120.

E-mail: ayokoyama@ntmc.hosp.go.jp

Key words: azacitidine; bridging to transplan; immunomodulater; acute GVHD.

Contributions: KM, designed the research, treated the patients, analyzed data, wrote and approved the manuscript; HU, designed the research, treated the patients, and approved the manuscript; TO, TK, SB, TY, treated the patients, and approved the manuscript; AY, designed the research, treated the patients, analyzed data, wrote and approved the manuscript.

Conflict of interest: the authors declare no potential conflict of interest.

Received for publication: 5 March 2017.

Revision received: 24 April 2017.

Accepted for publication: 9 May 2017.

This work is licensed under a Creative Commons Attribution-NonCommercial 4.0 International License (CC BY-NC 4.0).

CCopyright K. Murakami et al., 2017

Licensee PAGEPress, Italy

Hematology Reports 2017; 9:7114

doi:10.4081/hr.2017.7114

related donor, and tacrolimus with sMTX for alternative donor. All statistical analyses were performed with EZR. ${ }^{9}$

\section{Results}

Overall survival (OS) and event-free survival (EFS) were not significantly different between the two groups. Median OS was 202 days [ $95 \%$ confidence interval (CI), 80-NA] in the AZA group, and 458 days $(95 \% \mathrm{CI}, 53-\mathrm{NA})$ in the non-AZA group, respectively $(\mathrm{P}=0.941)$. Median EFS was 145 days $(95 \% \mathrm{CI}, 15-\mathrm{NA})$ in the AZA group, and 227.5 days $(95 \% \mathrm{CI}, 15-1237)$ in the non-AZA group, respectively $(\mathrm{P}=0.642)$. The events included death, acute and chronic GVHD, and relapse. No graft failure was seen in either group. It may not be relevant to compare the two groups directly because of their different backgrounds. However, one might assume that AZA may be considered as an effective therapy bridging between chemotherapy and pre-transplantation conditioning regi- 
mens. Two patients $(20 \%)$ of the AZA group, and 8 patients $(80 \%)$ of the nonAZA group developed grade II to IV acute GVHD (P=0.004). Only 1 patient $(10 \%)$ in the AZA group developed grade III to IV acute GVHD as compared to 5 patients $(50 \%)$ in the non-AZA group $(\mathrm{P}=0.04)$. Chronic GVHD occurred in 3 patients $(30 \%)$ in the AZA group and in 6 patients $(60 \%)$ in the non-AZA group; the difference was not statistically significant $(\mathrm{P}=0.30)$. The overall incidence of grade II to IV acute GVHD in the AZA group was significantly lower than that in the non-AZA group (data of grade III to IV acute GVHD are shown in Figure 1). The differences were not affected by death $(\mathrm{P}=0.004)$ and relapse $(\mathrm{P}=0.005)$ as competing risks of acute GVHD by FineGray competing risk regressions.

\section{Discussion and Conclusions}

There have been some retrospective studies addressing the impact of pre-transplantation therapy with AZA. Damaj et al. ${ }^{1}$ reported the effect of pre-transplant AZA on survival after allo-SCT for MDS. This is the largest study on pre-transplantation AZA therapy to date. In this study, 163 patients received cytoreductive treatment prior to transplantation, including ICT alone (ICT group, n=98), AZA alone (AZA group, $\mathrm{n}=48$ ), or AZA preceded or followed by ICT (AZA-ICT group, $\mathrm{n}=17$ ). With a median follow-up of 38.7 months, a-3-year outcome in the ICT, AZA, and AZA-ICT groups were $48 \%, 55 \%$, and $32 \%(\mathrm{P}=0.07)$ for OS; $44 \%, 42 \%$, and $29 \%(\mathrm{P}=0.14)$ for

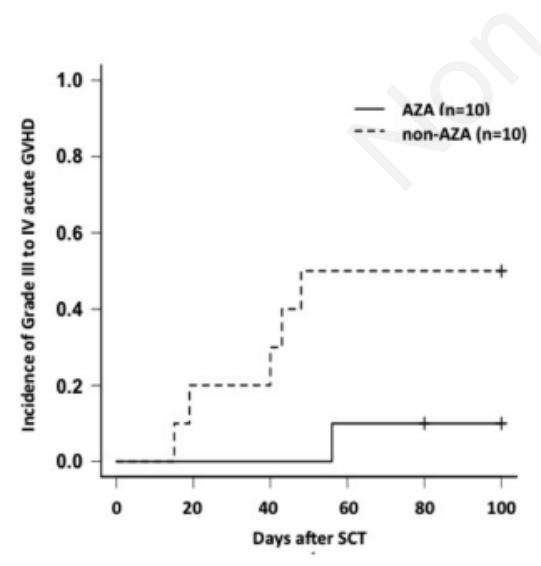

Figure 1. Incidence of Grade III to IV acute graft-versus-host disease (GVHD) after allogeneic stem cell transplantation (alloSCT) according to pretransplantation therapy: azacitidine (AZA) vs non-AZA. Patients were censored at the last followup. The difference between AZA and nonAZA was significant at $P=0.04$.
EFS, respectively. Multivariate analysis showed there were no statistically significant differences between the AZA and ICT groups in terms of OS and EFS. Our study has also showed a similar tendency on OS and EFS. The incidence rate of acute and chronic GVHD were not reported in study by Damaj et al.

Oshikawa et al. ${ }^{10}$ retrospectively reviewed clinical outcome of 15 MDS patients who were treated with AZA before allo-SCT (AZA group) compared with

52MDS patients who were solely given the best supportive care (BSC) before allo-SCT (BSC group). Although patients in the AZA group were older with higher IPSS scores compared to patients in the BSC group, no significant differences were found between the two groups in OS; 79.0 and $78.6 \%$, disease-free survival (DFS); 71.2 and $59.2 \%$, cumulative incidence of relapse; 15.4 and $11.3 \%$ or non-relapse mortality; 21.0 and $13.5 \%$, at 1 year. However, acute GVHD rate was similar between the two groups.

Table 1. Characteristics of participating patients and disease.

\begin{tabular}{|c|c|c|}
\hline & AZA Group (n) & Non-AZA Group (n) \\
\hline $\begin{array}{l}\text { Sex } \\
\quad \text { Male } \\
\quad \text { Female }\end{array}$ & $\begin{array}{l}6 \\
4\end{array}$ & $\begin{array}{l}7 \\
3\end{array}$ \\
\hline Age at SCT, years (mean) & $53(32-66)$ & $52.5(29-65)$ \\
\hline Days of follow-up & $314(80-1254)$ & $448(53-1314)$ \\
\hline Cycles of AZA & $3.5(1-6)$ & 0 \\
\hline $\begin{array}{l}\text { WHO classification } \\
\text { MDS } \\
\text { RCUD } \\
\text { RCMD } \\
\text { RAEB-1 } \\
\text { RAEB-2 } \\
\text { AML }\end{array}$ & $\begin{array}{l}8 \\
1 \\
4 \\
1 \\
2 \\
2\end{array}$ & $\begin{array}{l}3 \\
0 \\
3 \\
0 \\
0 \\
7\end{array}$ \\
\hline $\begin{array}{l}\text { IPSS } \\
\text { Low } \\
\text { Intermediate-1 } \\
\text { Intermediate-2 } \\
\text { High }\end{array}$ & $\begin{array}{l}0 \\
1 \\
6 \\
1\end{array}$ & $\begin{array}{l}0 \\
3 \\
0 \\
0\end{array}$ \\
\hline $\begin{array}{l}\text { IPSS-R } \\
\text { Very low } \\
\text { Low } \\
\text { Intermediate } \\
\text { High } \\
\text { Very high }\end{array}$ & $\begin{array}{l}1 \\
0 \\
1 \\
2 \\
4\end{array}$ & $\begin{array}{l}0 \\
2 \\
1 \\
0 \\
0\end{array}$ \\
\hline $\begin{array}{l}\text { Status at SCT } \\
\text { CR } \\
\text { PR } \\
\text { PIF }\end{array}$ & $\begin{array}{l}2 \\
3 \\
5\end{array}$ & $\begin{array}{l}5 \\
2 \\
3\end{array}$ \\
\hline $\begin{array}{l}\text { Donor } \\
\text { Related-PB } \\
\text { Unrelated-BM } \\
\text { Unrelated-CB }\end{array}$ & $\begin{array}{l}3 \\
6 \\
1\end{array}$ & $\begin{array}{l}6 \\
3 \\
1\end{array}$ \\
\hline $\begin{array}{l}\text { HLA compatibility } \\
8 / 8 \\
6 / 8 \\
5 / 8 \\
4 / 8\end{array}$ & $\begin{array}{l}8 \\
1 \\
1 \\
0\end{array}$ & $\begin{array}{l}7 \\
1 \\
1 \\
1\end{array}$ \\
\hline $\begin{array}{l}\text { Conditioning } \\
\text { MAC } \\
\text { RIC }\end{array}$ & $\begin{array}{l}4 \\
6\end{array}$ & $\begin{array}{l}5 \\
5\end{array}$ \\
\hline $\begin{array}{l}\text { HCT-CI } \\
0 \\
1-2 \\
3-4\end{array}$ & $\begin{array}{l}5 \\
2 \\
3\end{array}$ & $\begin{array}{l}6 \\
3 \\
1\end{array}$ \\
\hline
\end{tabular}

AML, acute myeloid leukemia; AZA, azacitidine; BM, bone marrow; CB, cord blood; CR, complete remission; HCT-CI, hematopoietic cell transplantation-comorbidity index; HLA, human leukocyte antigen; IPSS, International Prognostic Scoring System; IPSS-R, Revised International Prognostic Scoring System; MAC, myeloablative conditioning; MDS, myelodysplastic syndrome; PB, peripheral blood; PR, partial remission; PIF, primary induction failure; RAEB, refractory anemia with excess blasts; RCMD, refractory cytopenia of multilineage dysplasia; RCUD, refractory cytopenia of unilineage dysplasia; RIC, reduced-intensity conditioning; SCT, stem cell transplantation. 
There was no tissue injury before allo-SCT which will enhance GVHD in the control BSC group.

Gerds et $a l .{ }^{3}$ retrospectively reviewed their cases, reporting the incidence rate of acute and chronic GVHD. Thirty-five patients underwent allo-SCT for MDS or AML-MRC with AZA pre-transplantation therapy compared with 33 patients of the historical cohort who received ICT before allo-SCT. Grade III to IV acute GVHD developed significantly less in the AZA group $(\mathrm{P}=0.004)$. Sixty percent of the patients in the AZA group received reduced-intensity conditioning regimen, and all of the patients in the ICT group received high-dose conditioning regimen. One might argue that the difference in the incidence of acute GVHD might be affected by the conditioning regimen. However, our study suggested that despite its potential cytotoxic activity, pre-transplantation therapy with AZA could reduce acute GVHD. Our data also strongly suggested that AZA would be able to reduce acute GVHD since no significant differences were seen in conditioning regimen or donor type.

Several pre-clinical studies have shown the anti-GVHD effects of AZA. ${ }^{11,12}$ Cytokine genes are targets of multiple epigenetic mechanisms in $\mathrm{T}$ lymphocytes. AZA inhibits T-cell proliferation and activation, blocking cell cycle in the G0 to G1 phase and decreasing the production of proinflammatory cytokines such as tumor necrosis factor- $\alpha$ and interferon- $\gamma$, and leading to demethylation of FOXP3 promoter, overexpression of FOXP3, and expansion of Tregs.

These studies have shown the effect of AZA against $\mathrm{T}$ lymphocytes and elucidates its role in the allo-SCT setting as an immunomodulatory drug, describing new pathways that must be explored to prevent GVHD.

In our study, the AZA group showed higher $C D 4 / 8$ ratio after induction therapy than non-AZA group $[1.15 \pm 0.70(\mathrm{n}=9) v s$. $0.91 \pm 0.56(n=9)]$, but no significant difference was observed $(\mathrm{P}=0.22)$. This data seems to be supported by previous in vitro study that AZA treatment up-regulate CD4+ T-cells and down-regulate CD8+ T-cells. ${ }^{13}$ A recent study has also shown that, preemptive usage of AZA in patients at a high risk of relapse following allo-SCT, may help prevent/delay relapse. ${ }^{14}$ For patients who have a relapse following allo-SCT, AZA may be a salvage therapy option, either as monotherapy or in combination with donor lymphocyte infusions (DLI). ${ }^{15}$

Considering the lower rate of acute GVHD may be related to lessened tissue injury and mild toxicity profile, or a direct anti-GVHD effect of AZA. Bridging to transplant with AZA should be considered as a feasible and effective treatment strategy for patients with MDS and AML with myelodysplasia-related changes. Its effectiveness should be further investigated and validated by prospective randomized study.

\section{References}

1. Damaj G, Duhamel A, Robin M, et al. Impact of azacitidine before allogeneic stem-cell transplantation for myelodysplastic syndromes: a study by the Societe Francaise de Greffe de Moelle et de Therapie-Cellulaire and the Groupe-Francophone des Myelodysplasies. J Clin Oncol 2012;30: 4533-40.

2. Platzbecker U, Schetelig J, Finke J, et al. Allogeneic hematopoietic cell transplantation in patients age 60-70 years with de novo high-risk myelodysplastic syndrome or secondary acute myelogenous leukemia: comparison with patients lacking donors who received azacitidine. Biol Blood Marrow Transplant 2012;18:1415-21.

3. Gerds AT, Gooley TA, Estey EH, et al. Pretransplantation therapy with azacitidine vs induction chemotherapy and posttransplantation outcome in patients with MDS. Biol Blood Marrow Transplant 2012;18:1211-8.

4. Fenaux P, Mufti GJ, HellstromLindberg E, et al. Efficacy of azacitidine compared with that of conventional care regimens in the treatment of higher-risk myelodysplastic syndromes: a randomised, open-label, phase III study. Lancet Oncol 2009;10:223-32.

5. Dombret H, Seymour JF, Butrym A, et al. International phase 3 study of azacitidine vs conventional care regimens in older patients with newly diagnosed AML with $>30 \%$ blasts. Blood
2015;126:291-9.

6. Yamamoto R, Yokoyama A, Yoneda M, et al. Azacitidine as the post-remission therapy for elderly patients with acute myeloid leukemia. Ann Hematol 2014;93:2081-2.

7. Goodyear OC, Dennis M, Jilani NY, et al. Azacitidine augments expansion of regulatory $\mathrm{T}$ cells after allogeneic stem cell transplantation in patients with acute myeloid leukemia (AML). Blood 2012;119:3361-9.

8. Bashir Q, William BM, Garcia-Manero G, de Lima M. Epigenetic therapy in allogeneic hematopoietic stem cell transplantation. Rev Bras Hematol Hemoter 2013;35:126-33.

9. Kanda Y. Investigation of the freely available easy-to-use software EZR for medical statistics. Bone Marrow Transplant 2013;48:452-8.

10. Oshikawa G, Yoshioka K, Takahashi Y, et al. Impact of prior azacitidine on the outcome of allogeneic hematopoietic transplantation for myelodysplastic syndrome. Pathol Oncol Res 2015;21: 1037-43.

11. Sanchez-Abarca LI, Gutierrez-Cosio S, Santamaria C, et al. Immunomodulatory effect of 5-azacytidine (5-azaC): potential role in the transplantation setting. Blood 2010;115:107-21.

12. Choi J, Ritchey J, Prior JL, et al. In vivo administration of hypomethylating agents mitigate graft-versus-host disease without sacrificing graft-versusleukemia. Blood 2010;116:129-39.

13. Stubig T, Badbaran A, Luetkens T, et al. 5-azacytidine promotes an inhibitory $\mathrm{T}$ cell phenotype and impairs immune mediated antileukemic activity. Mediators Inflamm 2014;2014:418292.

14. Craddock C, Jilani N, Siddique S, et al. Tolerability and clinical activity of posttransplantation azacitidine in patients allografted for acute myeloid leukemia treated on the RICAZA trial. Biol Blood Marrow Transplant 2016;22:385-90.

15. Schroeder T, Czibere A, Platzbecker U, et al. Azacitidine and donor lymphocyte infusions as first salvage therapy for relapse of AML or MDS after allogeneic stem cell transplantation. Leukemia 2013;27:1229-35. 